\title{
Characterization of Metallic Core-Shell Structure of Electro-Catalysts
}

\author{
Dong Su,* Lijun Wu,** Hiromi Inada,**** Jia X. Wang,*** Weiping Zhou,*** Kotaro Sasaki,***
} Kuanping Gong,*** Radoslav R. Adzic,*** and Yimei Zhu,***

* Center for Functional Nanomaterials, ** Condensed Matter Physics and Materials Science Department, and *** Chemistry Department, Brookhaven National Laboratory, Upton, New York 11973

**** Nanotechnology Products Business Group, Hitachi High Technologies Corp, Ibaraki 3128504, Japan

Proton exchange membrane fuel cells (PEMFCs) convert the chemical energy of hydrogen to electricity with promising applications ranging from the electronics to automobile industries, and an efficient electrocatalysts for oxygen reduction reaction (ORR) play a decisive role in controlling the performance of the cathodes. High cost and low performance are two major obstacles related to ORR electro-catalysts that preclude the commercialization of PEMFCs[1]. The Pt-based core-shell structural electro-catalysts have been developed to reduce the cost and improve the oxygen reduction rate. Lattice contraction induced by size-effect and lattice mismatch between the Pt and core materials reduce the oxygen binding strength and therefore enhance the ORR activity. It is of very importance to address how the ORR activity varying with Pt shell thickness, nanoparticles sizes as well as core materials.

In this presentation, we demonstrate our electron microscopy investigation on Pt based core-shell catalysts in various core-shell structures: such as $\mathrm{Pd}\left(\right.$ core)-Pt(shell), $\operatorname{Pd}_{2} \mathrm{Co}$ (core)-Pt(shell), $\mathrm{Ir} / \mathrm{Ni}$ (core)-Pt(shell) et al. We mainly use two approaches based on scanning transmission electron microscopy (STEM): Z-contrast high angle annular dark field (HAADF) imaging and high resolution electron energy loss spectroscopy (EELS) spectrum imaging (1D or 2D). Figure 1 shows HAADF images taken on $\mathrm{Pd}_{\mathrm{C}} \mathrm{Pt}_{1}$ and $\mathrm{Pd}_{\mathrm{C}} \mathrm{Pt}_{4}$ nano-catalysts obtained using galvanic displacement of an underpotentially deposited (UPD) $\mathrm{Cu}$ layer method. The intensities of atomic columns are simulated based on core-shell structural models with different thickness [2]. Figure 2 shows $\mathrm{Pt} / \mathrm{Pd}_{2} \mathrm{Co}$ electrocatalysts with core-shell structure and the $0.5 \mathrm{~nm}$ thick Pt shell over $\mathrm{Pd}_{2} \mathrm{Co}$ core has been identified through our simultaneously recorded EELS and STEM signals [3]. Combining with in-situ X-ray absorption spectroscopy (XAS) study, we have retrieved the structural information of both one single nanoparticle and the entire catalyst samples. Our results indicate that the homogeneous and complete Pt shell is responsible for the high Pt mass activity and specific activity which is much higher than that of the state-of-art commercial Pt catalysts. The new insights gained from structural study may give clue for synthesizing high efficiency and low cost electro-catalysts. The practical issues of using the STEM imaging and EELS spectrum imaging techniques will be reviewed.

\section{Reference}

[1] R. R. Adzic, Recent Advances in the kinetics of oxygen reduction. In Electrocatalysis; J. Lipkowski, P. N. Ross, Eds.; Wiley: New York, 1998; pp 197.

[2] J. X. Wang; H. Inada, L. Wu, Y. Zhu, Y. Choi, P. Liu, W.-P. Zhou, R. R. Adzic, J. Am. Chem. Soc. 131 (2009) 17298. 
[3] Wei-Ping Zhou, Kotaro Sasaki, Dong Su, Yimei Zhu, Jia X. Wang, Radoslav R. Adzic, Unpublished.

[4] The work at Brookhaven was support by US Department of Energy BES, under contract No. DEAC02-98CH10886.
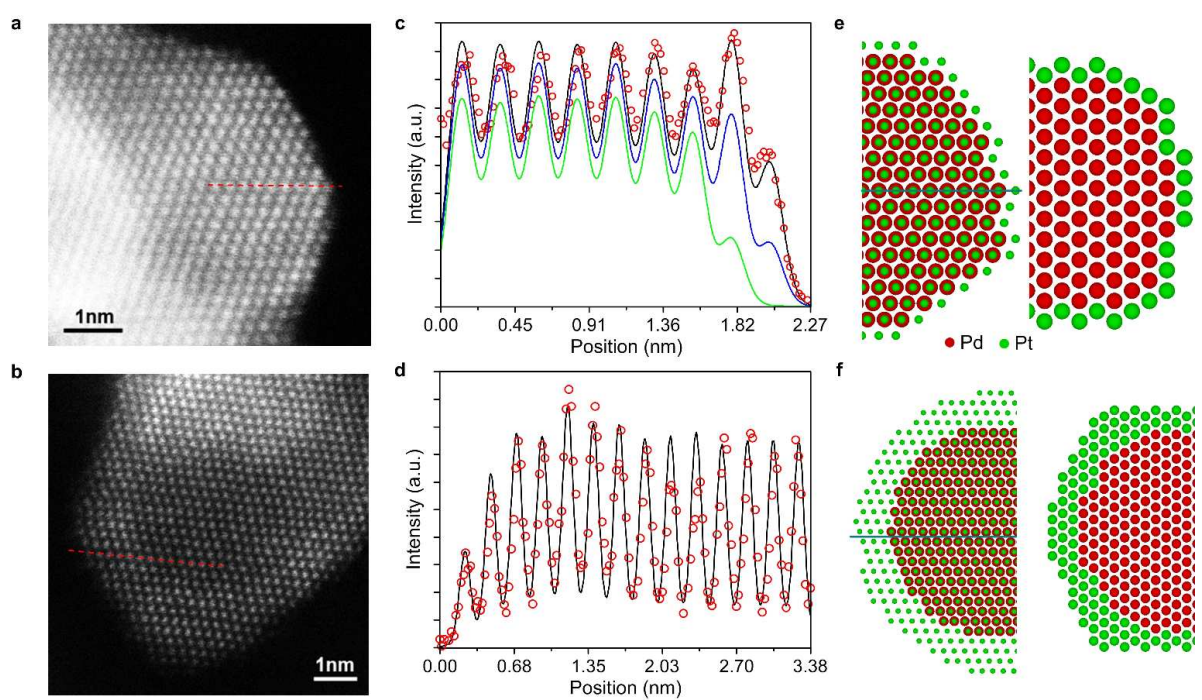

Figure 1 a,b, HAADF-STEM images of the Pd(core)-Pt(shell) nanoparticles obtained for the $\mathrm{Pd}_{\mathrm{C}} \mathrm{Pt}_{1}$ and $\mathrm{Pd}_{\mathrm{C}} \mathrm{Pt}_{4}$ samples, respectively. c,d, Intensity profiles from the scan lines in $\mathbf{a}$ and $\mathbf{b}$ (open circles), and the best fits (black lines), based on the structure models shown in $\mathbf{e}$ and $\mathbf{f}$ for Pd particles with 1 and 3-4 Pt surface layers, respectively. The calculations are convoluted with a Gaussian point spread function. Two additional lines in $\mathbf{c}$ show the calculations made with all the Pt surface atoms being removed (green line), or replaced by Pd (blue line). e, Projection of the one-Pt-layer core-shell structure model along the [101] direction (left), and the atomic arrangement (right) of the (1-1-1) plane at the position indicated by the line in the left. f, Projection of the structure model with $4 \mathrm{Pt}$ layers on the STEM image plane (left) and the arrangements of atoms in the vertical columns along the scan line (right).
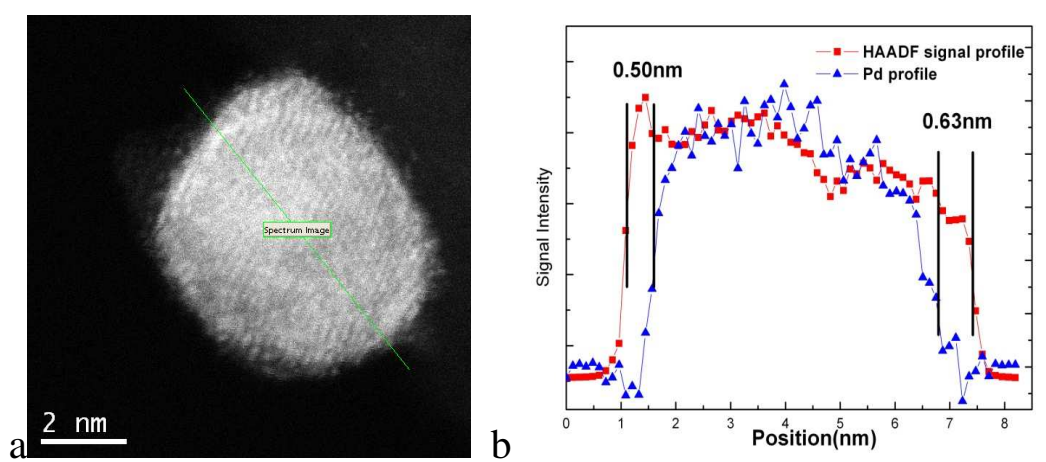

Figure 2: (a) Representative of HAADF-STEM for a $\mathrm{Pd}_{2} \mathrm{Co}$ (core)-Pt(shell) nanoparticle on carbonblack. (b) Comparison of the corresponding HAADF (red) and Pd M edge signal's (blue) intensity profiles from the EELS line scan indicated as a green line in (a). For the Pd EELS profile, we use an energy window of about $100 \mathrm{eV}$ to retrieve the signal. Both intensity profiles have been normalized to their maximum of intensity. 